\title{
Percepción de inseguridad ciudadana y su relación con la ideología política en una muestra de habitantes de la ciudad de Lima
}

\author{
Jan Marc Rottenbacher de Rojas, Laura Amaya López, Karen Genna \\ Miyahira, Maribel Pulache Páez \\ Pontificia Universidad Católica del Perú (PUCP). \\ e-mail: jrottenbacher@pucp.edu.pe
}

Original recibido el 4 de mayo de 2009

\begin{abstract}
RESUMEN
El presente estudio analiza la relación entre la percepción de inseguridad ciudadana, el miedo al delito, la ideología política y la frecuencia de victimización; en una muestra de habitantes de la ciudad de Lima. Como medidas de ideología política se utilizaron las escalas de autoritarismo de ala derecha (RWA) y de orientación hacia la dominancia social (SDO). Para la medición de la percepción de inseguridad ciudadana se desarrolló una escala ad hoc. Se encontró una correlación positiva entre el autoritarismo, la orientación hacia la dominancia social y la percepción de inseguridad ciudadana. Se encontró además, una correlación positiva entre el autoritarismo y la intensidad con la que se percibe el peligro. Los resultados sugieren que una mayor tendencia hacia el autoritarismo está asociada con la creencia de que la inseguridad y la delincuencia están vinculadas con los grupos de menor estatus social.
\end{abstract}

Palabras clave: percepción de inseguridad ciudadana; miedo al delito; victimización; ideología política; autoritarismo; dominancia social.

\begin{abstract}
The present study analyzes the relationship between perception of citizen's insecurity, fear of crime, political ideology and the frequency of victimization in a sample of Lima citizens. The Right Wing Authoritarianism Scale (RWA) and the Social Dominance Orientation Scale (SDO) were used as measures of political ideology. An ad hoc scale was developed to evaluate the perception of citizen's insecurity. It was found a positive correlation between perception of citizen's insecurity, authoritarianism and social dominance orientation. A positive correlation between authoritarianism and the intensity of risk perception was also found. A higher tendency to be authoritarian seems to be associated with the belief that insecurity and crime are related to lower status social groups.
\end{abstract}

Keywords: perception of citizen's insecurity; fear of crime; victimization; political ideology; authoritarianism; social dominance. 


\section{Introducción}

Uno de los principales problemas que afrontan actualmente las grandes ciudades de Latinoamérica es el elevado número de conductas delictivas y antisociales (Carrión, 2007). Como afirman algunos autores, este problema, junto con la ineficiencia de los organismos gubernamentales de seguridad interna, han contribuido al incremento de la percepción de inseguridad ciudadana (Muñoz \& Instituto de Defensa Legal (IDEELE), 2008; Basombrío, 2007). Sin embargo, estas percepciones suelen variar dependiendo, entre otros factores, de la experiencia previa de victimización (Mesch, 2000) y de la ideología política de cada individuo (Altemeyer, 1998, 2006; Duckitt, 2001; Jost, Glaser, Kruglanski \& Sullowey, 2003; Crowson \& Debacker, 2006). La ideología política como constructo psicológico comprende, entre otras, dos variables objeto de estudio en la presente investigación: el Autoritarismo de Derecha (RWA ${ }^{1}$ ) y la Orientación hacia la Dominancia Social (SDO²) (Biernat \& Crandall, 1999; Cottam, Dietz-Uhler, Mastors \& Preston, 2004; Crowson, Thoma \& Hestevold, 2005). Ambas resultan pertinentes para poder entender, de manera más específica, el fenómeno de la inseguridad ciudadana.

\section{Percepción de Inseguridad Ciudadana, Miedo al Delito y Victimización}

\section{Inseguridad ciudadana}

La percepción de inseguridad ciudadana constituye un concepto que corresponde al campo de las representaciones sociales e imaginarios colectivos (Carrión, 2007). Para Curbet (2006) la inseguridad ciudadana incluye dos componentes: la inseguridad subjetiva y la inseguridad objetiva. La primera se define como el sentimiento de amenaza personal, el cual no necesariamente corresponde con la vulnerabilidad real de la persona que la experimenta (Carrión, 2007). La segunda comprende un análisis objetivo de la probabilidad de ser víctima de algún delito (Dammert, 2005).

En una visión comparativa hecha por el Banco Interamericano de Desarrollo sobre la percepción del aumento de la delincuencia en Latinoamérica en 2006, el Perú se encontraba entre los países que presentaban una percepción más pesimista con respecto a este fenómeno (Basombrío, 2007). A pesar de ser uno de los países latinoamericanos

\footnotetext{
${ }^{1}$ RWA, de las siglas en inglés de Right Wing Authoritarianism.

${ }^{2}$ SDO, de las siglas en inglés de Social Dominance Orientation.
}

Revista Española de Investigación Criminológica

Artículo 4, Número 7 (2009) $\quad$ www.criminología.net

ISSN: $1696-9219$ 
con los índices más bajos de criminalidad en términos cuantitativos (Varat, 2007), las encuestas de percepción de inseguridad en el Perú han recogido la misma respuesta generalizada: la delincuencia en las calles ha aumentado con respecto al periodo precedente. Esto incluso a pesar de que el $64.1 \%$ de los encuestados manifiesta no haber sido víctima directa (Sistema Nacional de Seguridad Ciudadana (SINASEC ${ }^{3}$ ) \& Ipsos APOYO, Opinión y Mercado $\left.{ }^{4}, 2005\right)$.

En el Perú, la delincuencia y la falta de seguridad son consideradas como el segundo problema más importante del país después de la pobreza. Por otro lado, Lima es la ciudad con el mayor porcentaje de violencia delincuencial (Varat, 2007). Frente a esto, el $87 \%$ de la población se siente insegura cuando sale a la calle (Grupo de Opinión Pública de la Universidad de Lima, 2007). En otros estudios, el robo y el delito común son los crímenes que despiertan mayor temor en la ciudadanía y que además presentan la mayor tasa de frecuencia objetiva (SINASEC \& Ipsos APOYO, Opinión y Mercado, 2005; Instituto de Opinión Pública de la Pontificia Universidad Católica del Perú (IOP), 2008; Muñoz \& IDEELE, 2008). Según Yépez (2004), la sensación de seguridad se vio fuertemente afectada por la violencia terrorista en el Perú, donde el número de víctimas que es estimado por la población supera notoriamente las cifras oficiales. De acuerdo con estos estudios, podemos sugerir que la población percibe mayor inseguridad en comparación con el panorama que presentan las cifras oficiales sobre la delincuencia. Por otro lado, existe una diferencia considerable en la percepción de inseguridad según el nivel de proximidad del ámbito geográfico: el barrio, la ciudad y el país en el que vive la persona, son percibidos de manera diferente. Los entornos más próximos como el distrito o barrio de residencia se perciben menos inseguros que el país en general. Esta diferencia se presenta independientemente del estrato socioeconómico del sujeto. La inseguridad es percibida más como un problema de otros que de uno mismo (Basombrío, 2007). Finalmente, Curbet (2006), divide a la población según los recursos cognitivos con los que cuenta para enfrentarse a situaciones de peligro. De esta manera, la percepción de inseguridad se incrementa, no en aquellos directamente amenazados, sino en los sujetos con esquemas rígidos renuentes a los cambios sociales.

\footnotetext{
${ }^{3}$ En el Perú, en Sistema Nacional de Seguridad Ciudadana (SINASEC), forma parte del Consejo Nacional de Seguridad Ciudadana (CONASEC) que está bajo jurisdicción del Ministerio del Interior, entidad dependiente del gobierno central, que se encarga de la seguridad interna del país.

${ }^{4}$ El Grupo APOYO es una de las consultoras más importantes en materia de opinión pública en el Perú.
}

Revista Española de Investigación Criminológica

Artículo 4, Número 7 (2009) $\quad$ www.criminología.net

ISSN: $1696-9219$ 


\section{Miedo al delito}

Podemos diferenciar los conceptos de miedo al delito y percepción de inseguridad ciudadana, debido a que el primero hace referencia al sentimiento de temor frente a signos asociados a la delincuencia común, mientras que la percepción de inseguridad ciudadana puede abarcar el miedo hacia fenómenos no necesariamente vinculados a la delincuencia tradicional, como el terrorismo, el vandalismo o el pandillaje (Vozmediano, San Juan, \& Vergara, 2008). Según Weinrath, Clarke \& Forde (2007) los tres modelos teóricos más difundidos para entender el miedo al delito son: a) el modelo de la interpretación de riesgo, b) el modelo de la victimización indirecta y c) el modelo de la vulnerabilidad. El primer modelo se centra en aquellos estímulos del ambiente que pueden ser interpretados como signos o señales de delincuencia y que generan miedo. El segundo modelo pone énfasis en el efecto que ejercen los medios de comunicación y las experiencias de personas que han sido víctimas, sobre aquellas personas no víctimas que se consideran víctimas indirectas. El tercer modelo propone que aquellas personas que se perciben a sí mismas como más vulnerables son las que suelen presentar mayores niveles de miedo al delito (Taylor \& Hale, 1986; Ferraro, 1995; Weinrath, Clarke \& Forde, 2007). Esto último está relacionado con el hallazgo de que el género es el predictor más fuerte de miedo al delito, siendo las mujeres las que presentan mayor temor. Otras variables asociadas positivamente con el miedo al delito son la edad, la pertenencia a minorías étnicas y niveles educativos más bajos (Weinrath, Clarke \& Forde, 2007). Para Kohm (2009), el miedo al delito ejerce un efecto negativo sobre la calidad de vida, ya que restringe la movilidad personal, disminuye la sociabilidad y afecta la confianza mutua entre las personas.

\section{Victimización}

Para Cruz (1999) la victimización es la situación en la cual una persona es objeto del uso de la fuerza, lo que le produce un daño físico o psicológico. Según Zaldívar (2007), la victimización es definida como la expropiación o el abuso de una o más personas por sobre otra, como también, el resultado de un comportamiento antisocial contra un grupo o persona. Para los fines de este estudio, la victimización ha sido definida como el hecho de haber sido víctima de algún delito (uso de la fuerza, comportamiento antisocial, expropiación o abuso) en los últimos 12 meses. 
Con respecto a la frecuencia de victimización y percepción de inseguridad ciudadana, los individuos que han sido víctimas de algún delito suelen presentar una mayor sensación de temor, especialmente las mujeres (Mesch, 2000). No obstante, algunos autores señalan que la experiencia previa de victimización no necesariamente genera mayor inseguridad (Hammond \& Calhoun, 2007). Como ejemplo de ello, podemos mencionar el caso del Perú, donde los jóvenes que no tuvieron contacto directo con la violencia terrorista, presentaron un mayor temor hacia dicho fenómeno que las personas que sí lo sufrieron (Basombrío \& Prado, 2004).

\section{Ideología Política}

Autoritarismo de derecha

El autoritarismo de derecha, es definido como la sumisión hacia las autoridades establecidas, la cual es producto de una combinación de predisposiciones en los rasgos de personalidad y eventos que ocurren en la vida del individuo. Está compuesto por tres grandes constructos actitudinales: sumisión a la autoridad, agresión autoritaria y convencionalismo (Cottam et al., 2004; Altemeyer, 2006). La primera se define como un alto grado de sumisión a las autoridades legitimadas por la sociedad. La agresión autoritaria es una acción dirigida en contra de personas que se consideran merecedoras de sanción. Finalmente, el convencionalismo constituye un alto grado de adhesión a las convenciones sociales que se perciben de tal forma que deberían ser adoptadas por la totalidad de la sociedad (Knight, 1999, Cottam et al., 2004). El autoritarismo de derecha se encuentra asociado con actitudes prejuiciosas, preferencia por partidos políticos de derecha, mayor orientación hacia la ley y el orden (Sabucedo, 1996), actitudes favorables hacia la guerra y la agresión o el castigo corporal como forma de penalización por parte de las autoridades (Benjamin, 2006; James, 2006). Aunque el autoritarismo no es sinónimo de conservadurismo, existe una fuerte asociación entre ambos constructos. Crowson, Thoma \& Hestevold (2005) proponen que el autoritarismo, la rigidez cognitiva y la necesidad de poseer creencias simples, esquemáticas y completas acerca del mundo, influyen sobre el conservadurismo. Según Duckitt \& Sibley (2007), la vinculación del autoritarismo con estos últimos constructos lo relaciona indirectamente con la orientación hacia la dominancia social. 


\section{Orientación hacia la dominancia social}

La orientación hacia la dominancia social se refiere al grado en el que un individuo se encuentra a favor de las relaciones intergrupales desiguales, jerárquicas y orientadas hacia la dominancia por parte de uno de los grupos, sentando las bases para la formación de prejuicios y conservadurismo (Biernat \& Crandall, 1999; Cottam et al., 2004; Henry, Sidanius, Levin, \& Pratto, 2005; Leanne, Son Hing, Bobocel, Zanna, \& McBride, 2007). Asimismo, está asociada con la competitividad, el deseo por el poder, sentimientos de superioridad y una menor preocupación y compasión por los demás (Pratto \& Shih, 2000; Duckitt, 2001; Leanne, et al., 2007).

\section{Ideología política y percepción de inseguridad ciudadana}

Varios estudios han demostrado que aquellas personas que presentan un alto nivel de autoritarismo perciben el mundo como un lugar muy peligroso, en donde los otros constituyen una amenaza directa a la seguridad personal (Altemeyer, 1998; Duckitt, 2001; Jost et al., 2003; Crowson \& Debacker, 2006; Duckitt \& Sibley, 2007). Por otro lado, las personas que presentan un alto grado de dominancia social manifiestan actitudes negativas, no hacia grupos peligrosos, sino hacia aquellos que se perciben como posibles competidores en cuanto a superioridad, como es el caso de los grupos socialmente subordinados, que tienen menos poder y estatus (Duckitt, 2006). Asimismo, como sostiene Aguirre (2008), el abuso de autoridad implica el miedo pero también el desprecio hacia aquellos considerados no solo diferentes, sino también inferiores. Ello se confirmaría a través de las estadísticas que revelan que las causas atribuidas al incremento de la delincuencia, tanto en Latinoamérica como en el Perú, son el desempleo y la pobreza (Dammert, 2005). La pobreza es la causa atribuida principalmente por los niveles socioeconómicos altos y medios, denominados A y $\mathrm{B}^{5}$ (IOP, 2008). Por otro lado, según Duckitt \& Sibley (2007), tanto el autoritarismo de derecha como la dominancia social, se encontrarían asociados con el prejuicio étnico y con actitudes negativas hacia aquellos individuos que se oponen a las normas y valores oficiales que promueve la sociedad.

\footnotetext{
${ }^{5}$ La segmentación de la población en estratos socioeconómicos denominados con las letras A, B, C, D y E; fue iniciada por la consultora peruana Ipsos APOYO, Opinión y Mercado. Esta división socioeconómica de la población en la ciudad de Lima es la más utilizada dentro de los estudios de opinión en el Perú.
}

Revista Española de Investigación Criminológica

Artículo 4, Número 7 (2009) $\quad$ www.criminología.net

ISSN: 1696-9219 
Con base en todo lo revisado anteriormente, este trabajo se ha propuesto describir y analizar la relación entre la ideología política, la frecuencia de victimización y la percepción de inseguridad ciudadana en una muestra de habitantes adultos jóvenes de la ciudad de Lima.

\section{Método}

\section{Participantes}

Los participantes de esta investigación son 88 personas de ambos sexos entre 19 y 30 años de edad, de los cuales 38 son hombres y 50 son mujeres. Con respecto al nivel educativo, 71 participantes son estudiantes universitarios, mientras 16 ya han culminado sus estudios superiores. Todos viven en la ciudad de Lima y pertenecen a los estratos socioeconómicos medio y medio alto.

\section{Procedimiento}

Se contactó a los participantes de manera individual en el campus universitario de la Pontificia Universidad Católica del Perú. Luego de obtener el consentimiento de los participantes, se les entregó el cuestionario de carácter autoaplicado según la disponibilidad particular de cada uno, indicándoles que respondan con la mayor veracidad posible según sus propias creencias o actitudes. Se les indicó además, el carácter anónimo de la encuesta. La información fue recogida entre los meses de mayo y junio de 2008 .

\section{Instrumentos}

El cuestionario utilizado incluye cuatro instrumentos además de una ficha de datos sociodemográficos:

\section{a) Percepción de Inseguridad Ciudadana}

Para la medición del constructo Percepción de Inseguridad Ciudadana se utilizó, en primer lugar, la Escala de afrontamiento frente a la percepción de peligro ${ }^{6}$. Esta escala fue considerada pertinente para esta investigación con base en la revisión conceptual, por lo que posee, según Kerlinger \& Lee (2002), validez de contenido. Está compuesta

\footnotetext{
${ }^{6}$ Esta escala fue diseñada y propuesta en el año 2006 por Agustín Espinosa Pezzia, profesor del Departamento de Psicología de la Pontificia Universidad Católica del Perú. De los 21 ítems que propuso Espinosa, 19 fueron seleccionados para utilizarlos en la presente investigación.
}

Revista Española de Investigación Criminológica

Artículo 4, Número 7 (2009) $\quad$ www.criminología.net

ISSN: $1696-9219$ 
de 19 ítems, que recogen la intención de las personas de evitar una posible exposición al peligro. Las opciones de respuesta de los ítems están en una escala tipo Likert de 4 puntos donde 1 = "Totalmente en desacuerdo" hasta 4 = "Totalmente de acuerdo". La confiabilidad general del instrumento es alta $(\alpha=.798)$ y posee una estructura factorial aceptable $(K M O=.653)$, lo que respalda su utilización en el presente estudio. En segundo lugar, se utilizó la Escala de Miedo al Delito, empleada inicialmente por Vozmediano, San Juan, \& Espinosa (2006) y validada posteriormente por Vozmediano, San Juan, \& Vergara (2008). En esta escala se presentan 12 delitos y se les pide a los participantes que respondan a la siguiente pregunta: ¿Con qué frecuencia, a lo largo de una semana, le preocupa ser víctima de los delitos que presentamos? La opción de respuesta es una escala tipo Likert de 3 puntos donde $0=$ "Nunca", 1 = " 1 ó 2 veces por semana" y 2 = "Casi a diario / A diario". En tercer lugar, se incluyó una escala de 4 preguntas que abordan la percepción de peligrosidad en cuatro ámbitos geográficos distintos: el Perú en general, la ciudad de Lima, el distrito y el barrio del participante. La escala Likert es de 10 puntos donde $1=$ "Nada peligroso" y $10=$ "Muy peligroso". Finalmente, se consideraron dos preguntas que aluden a la probabilidad percibida de ser víctima de un delito en los próximos 12 meses, así como la probabilidad percibida de que el limeño promedio lo sea. La opción de respuesta comprende desde $0 \%=$ "Ninguna probabilidad" hasta $100 \%=$ "Total probabilidad".

\section{b) Victimización}

Para medir la frecuencia de victimización se utilizó la escala de victimización empleada inicialmente por Vozmediano, San Juan, \& Espinosa (2006). En ella se presentan los mismos 12 delitos de la Escala de Miedo al Delito y se le pide a la persona que responda "Sí” o "No" a la pregunta: Durante el último año, ¿Ha sido víctima en alguna ocasión de estos delitos? Se consideró como víctima a la persona que marcó "Sí” en por lo menos uno de los 12 delitos.

\section{c) Ideología política}

Como medida de ideología política se utilizó, en primer lugar, la versión traducida al castellano realizada por Moya \& Morales-Marente (2005) de la Escala de Autoritarismo de Ala Derecha (Right Wing Authoritarianism, RWA de Altemeyer). Esta escala comprende 32 enunciados que se agrupan en una sola área relacionada con creencias

Revista Española de Investigación Criminológica

Artículo 4, Número 7 (2009) $\quad$ www.criminología.net

ISSN: $1696-9219$ 
conservadoras y autoritarias. La escala de respuesta es de tipo Likert de 9 puntos en la que: -4 = "Muy fuertemente en desacuerdo" y 4 = "Muy fuertemente de acuerdo". El nivel de confiabilidad de este instrumento resultó alto para el caso de la muestra ( $\alpha$ $=.780$ ). En segundo lugar, se utilizó la versión traducida al castellano por MontesBerges \& Silva-Ferrero (citados en Moya \& Morales-Marente, 2005) de la Escala de Orientación hacia la Dominancia Social (Social Dominance Orientation, SDO de Sidanius y Pratto). Esta escala comprende 16 enunciados referidos a relaciones jerárquicas y asimétricas entre grupos pertenecientes a una misma sociedad. Los ítems están presentados en una escala tipo Likert de 7 puntos en la que $1=$ "Totalmente en desacuerdo" y 7 = "Totalmente de acuerdo". El nivel de confiabilidad de este instrumento resultó alto para el caso de la muestra $(\alpha=.845)$.

\section{Resultados}

Análisis factorial de la Escala de afrontamiento frente a la percepción de peligro Se realizó un análisis factorial exploratorio debido a que la Escala de afrontamiento frente a la percepción de peligro presentó una estructura factorial aceptable $(K M O=$ .653). A partir de este análisis se obtuvo 4 factores que explican el $55.39 \%$ de la varianza total y cuya descripción aparece en la siguiente tabla: 
Tabla 1

Factores de la Escala de afrontamiento frente a la percepción de peligro y los ítems asociados a ellos

\begin{tabular}{|c|c|c|c|c|}
\hline Ítems & F1 & $\mathrm{F} 2$ & F3 & $\mathrm{F} 4$ \\
\hline Evito tomar taxi solo/a & .919 & & & \\
\hline Evito tomar taxi de noche & .901 & & & \\
\hline Evito caminar por la calle de noche & .777 & & & \\
\hline Evito salir a la calle de noche & .669 & & & \\
\hline Evito sacar dinero de los cajeros automáticos durante la noche & .586 & & & \\
\hline Evito ir a zonas urbano-marginales & & .829 & & \\
\hline Trato de no relacionarme con gente pobre & & .749 & & \\
\hline La delincuencia es un problema originado por los pobres & & .736 & & \\
\hline Siempre estoy atento para ver si alguien me sigue & & & .720 & \\
\hline Me traslado por zonas y rutas transitadas por mucha gente & & & .698 & \\
\hline Procuro cerrar bien las ventanas y puertas de mi casa cuando salgo & & & .516 & \\
\hline Creo que la delincuencia ha obligado a los ciudadanos a encerrarse en sus barrios & & & .499 & \\
\hline Desconfío de la gente en la calle & & & .473 & \\
\hline Procuro cerrar bien las ventanas y puertas de mi casa cuando estoy en ella & & & .439 & \\
\hline No creo que me vaya a pasar algo malo en Lima (Recodificado) & & & .340 & \\
\hline Evito caminar por la calle de día & & & & .731 \\
\hline Evito sacar dinero de los cajeros automáticos durante el día & & & & .704 \\
\hline Evito salir a restaurantes & & & & .633 \\
\hline Evito salir a la calle de día & & & & .589 \\
\hline
\end{tabular}

Los cuatro componentes obtenidos del análisis factorial han sido denominados de la siguiente manera según los ítems asociados a ellos:

Factor 1 (F1): Afrontamiento evitativo prudente. Este factor agrupa 5 ítems que se refieren a conductas evitativas para situaciones que normalmente pueden resultar peligrosas en una ciudad como Lima. Este factor presentó un nivel de confiabilidad alto para el caso de la muestra $(\alpha=.857)$.

Factor 2 (F2): Evitación de grupos sociales en situación de pobreza. Este factor agrupa 3 ítems que asocian la delincuencia y el peligro que esta supone, con un grupo de menor estatus social denominado "los pobres" o "la gente pobre". Este factor presentó un nivel de confiabilidad alto para el caso de la muestra $(\alpha=.762)$.

Factor 3 (F3): Afrontamiento activo preventivo. Este factor agrupa 7 ítems relacionados a conductas de afrontamiento activo (no evitativo) con respecto al peligro percibido. Asimismo, los ítems de este factor describen un estado de alerta con respecto a la 
posibilidad de estar frente a una situación de peligro. Este factor presentó un nivel de confiabilidad aceptable ${ }^{7}$ para el caso de la muestra $(\alpha=.621)$.

Factor 4 (F4): Afrontamiento evitativo extremo. Este factor refleja que la persona percibe un alto grado de peligro en el ambiente urbano que la lleva a evitar situaciones cotidianas normalmente no peligrosas. Este factor presentó un nivel de confiabilidad aceptable para el caso de la muestra $(\alpha=.659)$.

Tabla 2

Correlaciones no paramétricas entre las variables de Ideología Politica y los factores de la Escala de afrontamiento frente a la percepción de peligro

\begin{tabular}{|c|c|c|c|c|c|c|}
\hline & $R W A$ & $S D O$ & AEP & EvPob & AAP & $\mathrm{AEE}$ \\
\hline$R W A$ & ----- & $.467 * *$ & .057 & $.256^{*}$ & .147 & $.268^{* *}$ \\
\hline$S D O$ & & ----- & -.153 & $.234^{*}$ & .032 & .108 \\
\hline Afrontamiento evitativo prudente (AEP) & & & ------ & $.184^{*}$ & $.286^{* *}$ & $.308^{* *}$ \\
\hline $\begin{array}{l}\text { Evitación de grupos sociales en situación de pobreza } \\
\text { (EvPob) }\end{array}$ & & & & ------ & $.223^{*}$ & .174 \\
\hline Afrontamiento activo preventivo (AAP) & & & & & ------ & $.223^{*}$ \\
\hline Afrontamiento evitativo extremo (AEE) & & & & & & ----- \\
\hline $\begin{array}{l}* * p \leq .01 \\
* p \leq .05\end{array}$ & & & & & & \\
\hline
\end{tabular}

En la tabla 2, se observa que las escalas RWA y SDO presentan entre ellas una correlación positiva de magnitud mediana. Ambas escalas presentan correlaciones positivas de magnitud pequeña pero significativa con el factor Evitación de grupos sociales en situación de pobreza, mientas que el RWA presenta una correlación ligeramente mayor con el factor Afrontamiento evitativo extremo.

\footnotetext{
${ }^{7}$ La confiabilidad se consideró aceptable si el coeficiente Alpha de Cronbach es mayor a .60, según los criterios propuestos por Cohen (1988).
}

Revista Española de Investigación Criminológica

Artículo 4, Número 7 (2009) $\quad$ www.criminología.net

ISSN: $1696-9219$ 
Tabla 3

Correlaciones no paramétricas entre las variables de Ideología Política y el grado de peligrosidad en los diferentes ámbitos geográficos

\begin{tabular}{|c|c|c|c|c|c|c|}
\hline & $R W A$ & $S D O$ & PelPerú & PelLima & PelDist & PelBarr \\
\hline$R W A$ & ------ & $.467 * *$ & -.102 & .035 & .122 & $.186^{*}$ \\
\hline$S D O$ & & ----- & -.051 & -.067 & $-.240 *$ & $-.206^{*}$ \\
\hline Peligrosidad en el Perú (PelPerú) & & & ------ & $.643^{* *}$ & .146 & .128 \\
\hline Peligrosidad en Lima (PelLima) & & & & ------ & $.303 * *$ & $.196^{*}$ \\
\hline Peligrosidad en su Distrito (PelDist) & & & & & ------ & $.802 * *$ \\
\hline Peligrosidad en su Barrio (PelBarr) & & & & & & ------ \\
\hline $\begin{array}{l}* * p \leq .01 \\
* p \leq .05\end{array}$ & & & & & & \\
\hline
\end{tabular}

En la tabla 3, se observa que la escala SDO correlaciona con una intensidad pequeña pero significativa y de manera inversa con la percepción de peligrosidad en el distrito y en el barrio de residencia. Las percepciones de peligro en el distrito y en el barrio de residencia correlacionan entre ellas de manera alta y positiva. La correlación entre la percepción de peligro en Lima y el Perú en general es también positiva aunque ligeramente menor.

\section{Frecuencia de victimización}

De la muestra total, 48 personas $(55.6 \%)$ reportaron haber sido víctimas de por lo menos un delito en los últimos 12 meses. El 44.4\% restante menciona no haber sido víctima de ningún delito, en el mismo periodo. Dos personas no respondieron esta pregunta. 


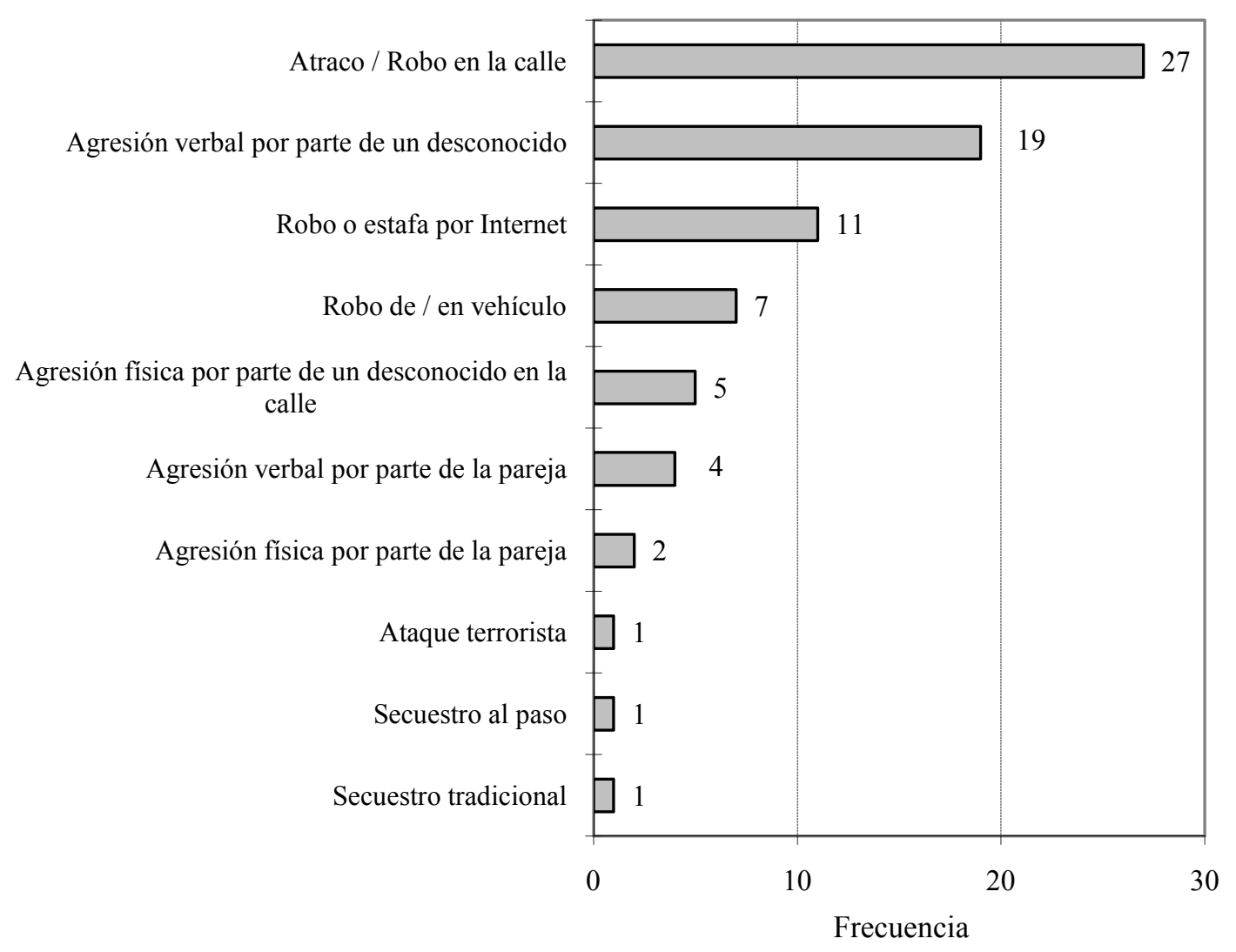

En el gráfico 1, aparecen los diez delitos que presentaron una frecuencia de mención mayor o igual a uno. El delito más frecuentemente mencionado es el atraco o robo en la calle $(n=27)$. En segundo lugar, se encuentra la agresión verbal por parte de un desconocido $(n=19)$ y en tercer lugar el robo o estafa por Internet $(n=11)$.

\section{Diferencias entre víctimas de algún delito y no víctimas}

No se encontraron diferencias significativas entre víctimas y no víctimas con respecto a ningún factor de la Escala de afrontamiento frente a la percepción de peligro. Sin embargo, las personas que han sido víctimas perciben mayor peligrosidad en su distrito $(\bar{X}=5.50, D E=1.99)$ que las que no han sido víctimas $(\bar{X}=4.51, D E=2.02, U=$ $612.5, Z=-2.208, p=027)$. 
Gráfico 2

Percepción de peligrosidad por ámbito geográfico

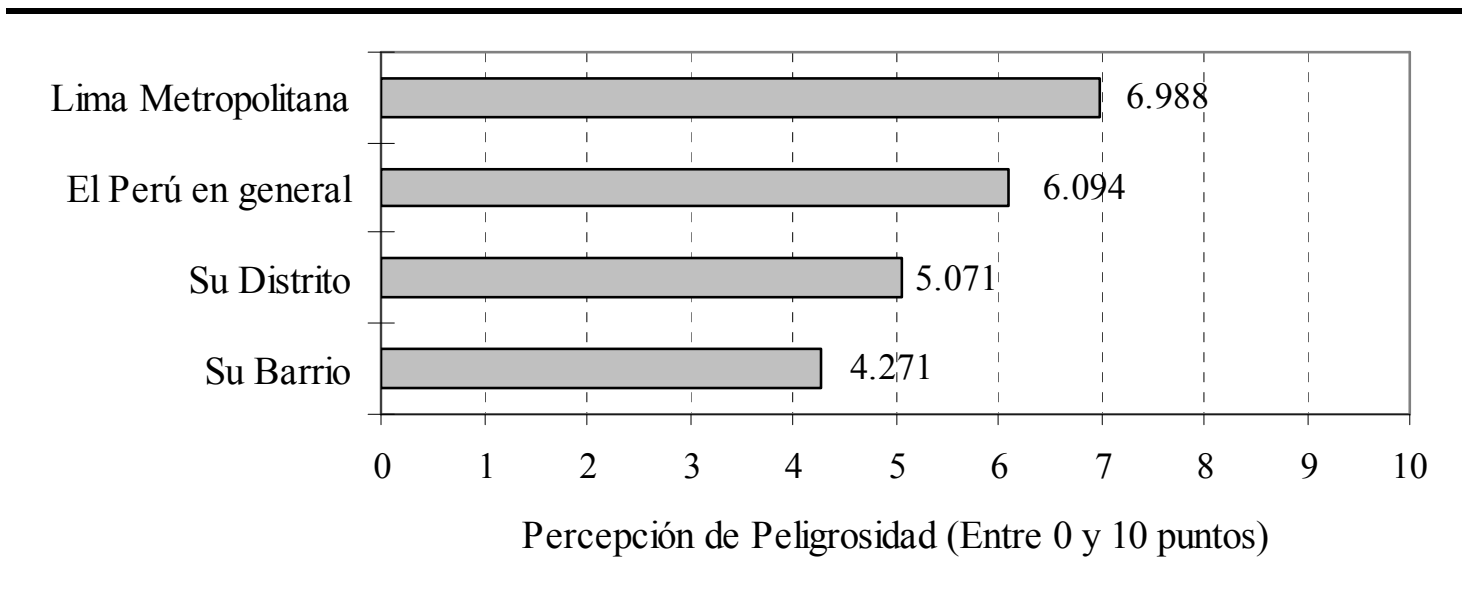

A nivel muestral $(N=88)$, el ámbito geográfico que se percibe como más peligroso es la ciudad de Lima. En segundo lugar se encuentra el Perú en general, en tercer lugar el distrito $y$, finalmente, el barrio de residencia del participante.

\section{Gráfico 3}

Revista Española de Investigación Criminológica

Artículo 4, Número 7 (2009) $\quad$ www.criminología.net

ISSN: 1696-9219 


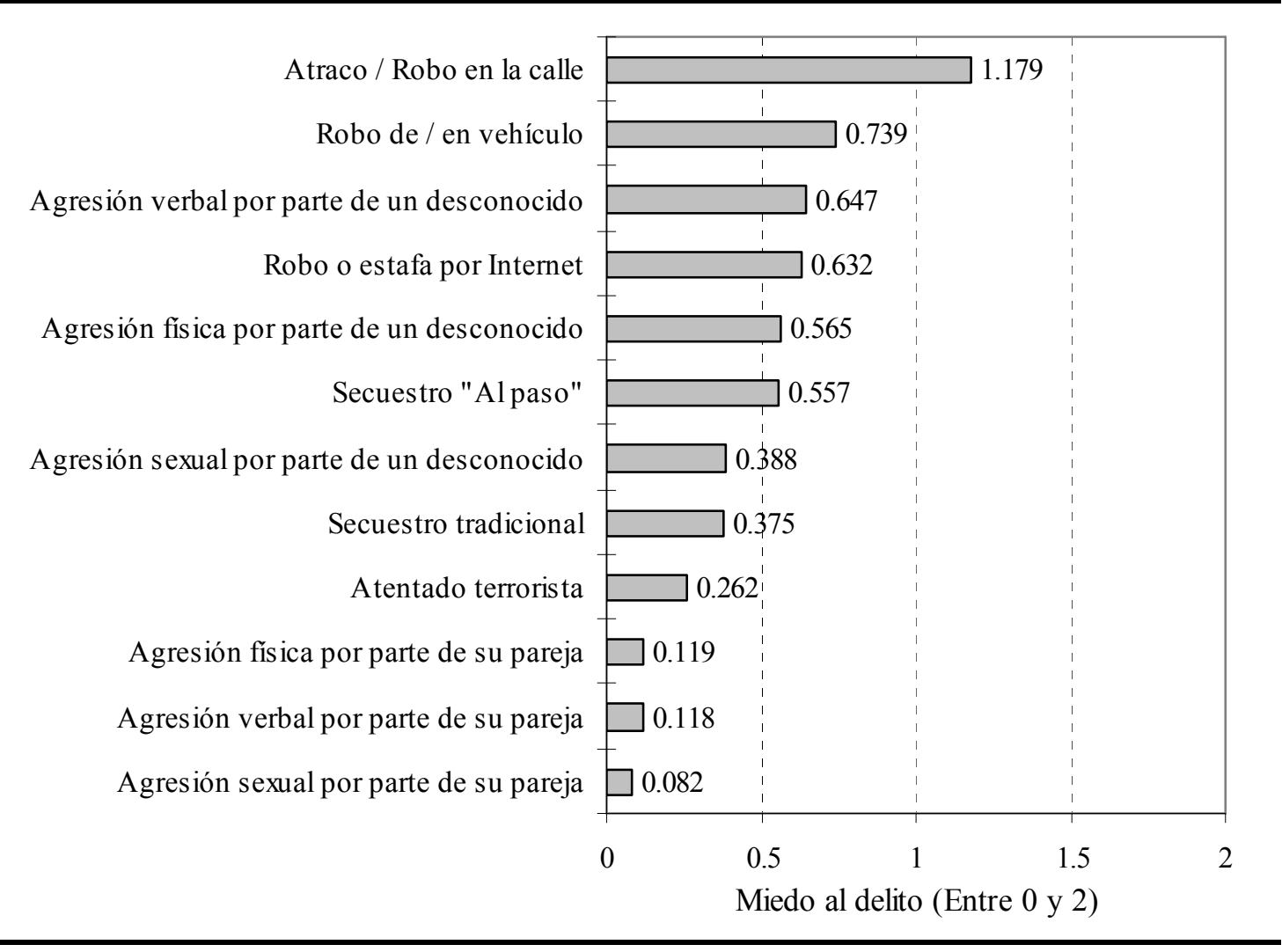

En el gráfico 3, se observa que el delito que genera mayor temor en los participantes es el robo en la calle. Otros delitos que también les preocupan a los participantes, aunque en menor medida, son: el robo del vehículo o en el vehículo, la agresión verbal de un desconocido en la calle, el robo o la estafa por Internet, la agresión física por parte de un desconocido y el denominado secuestro "al paso". La agresión por parte de la pareja, ya sea verbal, física o sexual son los delitos que menos preocupan a los participantes.

\section{Probabilidad percibida de ser víctima de algún delito}

En promedio, las personas atribuyen un mayor porcentaje de probabilidad de ser víctimas de delitos al "limeño promedio" (62.4\%. de probabilidad) que a sí mismos (44.2\% de probabilidad). 


\section{Diferencias por género}

Sólo se encontró una diferencia significativa entre hombres y mujeres con respecto a los factores de la escala de Escala de afrontamiento frente a la percepción de peligro. Las mujeres puntúan más alto en el factor denominado Afrontamiento evitativo prudente ( $\bar{X}=2.567, D E=0.626)$, en comparación con los hombres $(\bar{X}=1.840, D E=0.712, U=$ $397.5, Z=-4.459 p=.000)$.

\section{Discusión}

La presente investigación, posee algunas limitaciones metodológicas importantes, en especial, el tamaño reducido de la muestra y su grado limitado de representatividad con respecto a la población de la ciudad de Lima. Pese a esto, los resultados proponen indicios pertinentes para guiar futuras investigaciones de mayor envergadura. Para interpretar los resultados es necesario tomar en cuenta las limitaciones del estudio con respecto a la generalización estadística de los datos obtenidos.

En términos generales, se puede sugerir que en relación a la inseguridad ciudadana pueden existen dos planos estrechamente vinculados. Como afirma Curbet (2006), existiría un plano objetivo, es decir, que el peligro relacionado con la delincuencia en una ciudad como Lima es, en gran medida, real. Esto se observa en los resultados, cuando el $55.6 \%$ de la muestra manifestó haber sido víctima de por lo menos algún delito en los últimos doce meses. Por otro lado, existe un plano subjetivo, es decir, el conjunto de creencias y sentimientos asociados al peligro percibido y a sus posibles causas. Tomando en consideración la coexistencia de ambos planos se ha propuesto la presente discusión.

De acuerdo con los resultados obtenidos, la relación existente entre el autoritarismo de derecha y la orientación hacia la dominancia social, sugiere que estas son variables afines que reflejan la ideología política de los participantes del estudio. En este sentido, la ideología política se puede entender como un continuo, cuyos extremos estarían conformados por el polo conservador (personas más autoritarias y más orientadas hacia la dominancia social) y el polo liberal o progresista (personas menos autoritarias y con menor orientación hacia la dominancia social). 
Por otra parte, es importante señalar que el instrumento denominado Escala de afrontamiento frente a la percepción de peligro ha permitido identificar cuatro formas de afrontar la percepción de inseguridad ciudadana: Afrontamiento evitativo prudente, Evitación de grupos sociales en situación de pobreza, Afrontamiento activo preventivo y Afrontamiento evitativo extremo. Estas formas o estrategias de afrontamiento también sugieren la presencia de diferentes grados en cuanto al nivel de inseguridad percibido. Se ha encontrado una asociación positiva entre la Evitación de grupos sociales en situación de pobreza y la ideología política, lo que sugiere que personas más autoritarias y con mayor tendencia a aceptar la existencia de relaciones jerárquicas y desiguales entre los grupos sociales, perciben que los grupos menos privilegiados y de menor estatus (los llamados "pobres") forman parte de las causas de la delincuencia y por lo tanto, de la inseguridad ciudadana. En este sentido, "los pobres" son aquellos que se perciben como personas peligrosas que se debe evitar, reafirmando así el estereotipo del pobre-marginal-delincuente. Por un lado, como sostiene Aguirre (2008), el autoritarismo puede promover tanto el miedo como el rechazo hacia aquellos que se considera tanto diferentes como inferiores a uno mismo. Por otro lado, esto coincide con lo encontrado en uno de los estudios de opinión del IOP (2008): los estratos socioeconómicos medios y altos de Lima Metropolitana perciben a la pobreza como una de las causas de la delincuencia. La muestra del presente estudio pertenece a estos estratos socioeconómicos.

Es importante notar que el RWA está asociado de manera positiva al Afrontamiento evitativo extremo, lo que sugiere que las personas más autoritarias son las que perciben mayor grado de peligro, como lo han propuesto investigaciones previas (Duckitt, 2001; Jost, et al., 2003; Crowson \& Debacker, 2006; Duckitt \& Sibley, 2007). Con respecto a los tipos de delito de los cuales los participantes han sido víctimas, se encontró que el atraco o robo es el más frecuente dentro de la muestra. Esto corrobora lo encontrado en las numerosas encuestas sobre victimización en el Perú (SINASEC \& Ipsos APOYO, Opinión y Mercado, 2005). En el segundo lugar de frecuencia, se encontró la agresión verbal por parte de un desconocido y, en tercer lugar, el robo o estafa por Internet. Esto último, resulta muy llamativo y podría estar relacionado al aumento en el número de robos o estafas vía Internet que, según la empresa de software SYMANTEC (2004), se triplicó entre los años 2004 y 2005. También es posible sugerir que como los participantes pertenecen a generaciones jóvenes que utilizan la Red para

Revista Española de Investigación Criminológica 
una variedad de fines, incluyendo las transacciones financieras, están más expuestos a sufrir robos o estafas vía Internet. En general, como afirma Varat (2007), las denuncias por delitos graves y crímenes han disminuido, y delitos "leves" como los que se ha mencionado han presentado un aumento significativo en los últimos años.

Con respecto a la percepción de peligrosidad por ámbito geográfico, el entorno que se percibe como más peligroso es la ciudad de Lima. En segundo lugar, se encuentra el país en general, luego el distrito y finalmente, el propio barrio de residencia. Esto corrobora los hallazgos previos de Basombrío (2007), quien sostiene que los ambientes más cercanos tienden a percibirse como menos peligrosos. No obstante, que la ciudad de Lima se perciba más peligrosa que el país, puede estar relacionado con el dato objetivo que señala a Lima como la ciudad que concentra en el Perú, el mayor porcentaje de violencia delincuencial (Varat, 2007).

Otro resultado importante de mencionar es que las personas con mayor orientación hacia la dominancia social percibirían menores niveles de peligrosidad en su distrito y barrio de residencia. Esto se puede explicar debido a que estas personas suelen defender una posición privilegiada del endogrupo por sobre otros grupos sociales. La reivindicación del ambiente geográfico cercano como ambiente seguro, generaría en ellas la confirmación de sus creencias acerca de la jerarquización de la sociedad y del estatus privilegiado de su propio grupo de pertenencia.

En el ámbito subjetivo de la inseguridad ciudadana, el robo es el delito más temido entre los participantes, lo cual corrobora lo propuesto por Muñoz \& IDEELE (2008), quien afirma que el "robo común" es uno de los delitos que despierta mayor temor en la ciudadanía. El robo de o en el vehículo y el secuestro al paso también se encuentran en la lista de los delitos más temidos. Según SINASEC \& Ipsos APOYO, Opinión y Mercado (2005) existe un aumento de estos delitos en la ciudad de Lima, por lo que el plano objetivo de la inseguridad podría estar influyendo nuevamente en la percepción de los participantes.

Con respecto a la relación entre victimización y las demás variables de estudio, se encontró que los individuos con experiencia previa de victimización presentan una mayor percepción de riesgo incluso en uno de los ámbitos geográficos más cercanos como es su propio distrito de residencia. El lugar de victimización no ha sido indagado en esta investigación, pero es muy probable que el delito del cual fueron víctimas los participantes haya ocurrido en el distrito de residencia. 
En cuanto a la probabilidad de ser víctima, los resultados sugieren que los participantes perciben mayores probabilidades de peligro para el "limeño promedio" que para sí mismos. Esto se relaciona con lo que sostiene Basombrío (2007), que la inseguridad es percibida más como un problema de "otros", que de uno mismo. Finalmente, las mujeres puntúan más alto en el área denominada Afrontamiento evitativo prudente en comparación con los hombres. Este resultado coincide con los hallazgos de Weinrath, Clarke \& Forde (2007) en los que se afirma que el género es una de las variables más fuertemente relacionadas con el miedo al delito y el sentimiento de inseguridad. Esto podría deberse sencillamente, a lo que hemos denominado el plano objetivo: las mujeres serían más vulnerables y por lo tanto existe una mayor probabilidad de que sean víctimas de actos delictivos en una ciudad como Lima. Asimismo, como sostiene Ferraro (1995), la propia percepción de vulnerabilidad incrementa la sensación de inseguridad frente a la delincuencia. Sin embargo, según Ramos (2006), esta explicación resulta insuficiente, pues propone que los hombres son, en general, la mayoría de las víctimas de los actos delictivos como el robo o asalto. Este autor sugiere que según las creencias que sustentan algunos roles de género en el Perú, las mujeres deberían exponerse menos al peligro que los hombres, debido en parte, a una vulnerabilidad objetiva. Por otro lado, este autor afirma que el concepto de masculinidad más difundido entre los varones limeños, incluye la creencia de que el hombre puede y hasta debe, enfrentarse a situaciones peligrosas en defensa de su propia masculinidad. La afirmación de la masculinidad podría estar a la base, de la exposición de los hombres a mayores situaciones de riesgo y de la protección que éstos deben ofrecer a las mujeres, según los roles de género predominantes (Ramos, 2006, 2008).

Se sugiere que hay indicios suficientes para considerar pertinente la relación entre la ideología política, el miedo al delito y la percepción de inseguridad ciudadana. Este es un campo de estudio que debería ser estudiado con mayor profundidad a partir de investigaciones con un grado mayor de representatividad estadística. 


\section{Referencias}

Aguirre, C. (2008). Dénle duro que no siente: Poder y transgresión en el Perú republicano. Lima: AFINED.

Altemeyer, B. (1998). The other authoritarian personality. En Zanna, M. (Ed.), Advances in Experimental Social Psychology, 30, 47-92. San Diego: Academic Press.

(2006). Highly Dominating, Highly Authoritarian Personalities. The Journal of Social Psychology, 144, 4, 421-447.

Basombrío, C. (2004). Seguridad ciudadana y actuación del Estado: Análisis de Las tendencias de opinión pública. Lima: Bellido Ediciones.

----------. (2007). Lima y otras ciudades del Perú comparadas con América Latina. Lima: Bellido Ediciones.

Basombrío, C. \& Prado, G. (2004). Manejo y gestión de la seguridad. Lima: Instituto de Defensa Legal.

Benjamin, A. J., (2006). The relationship between Right-Wing Authoritarianism and Attitudes toward Violence: Further validation of the Attitudes toward Violence Scale. Social Behavior And Personality, 34, 923-926.

Biernat, M. \& Crandall, C. (1999). Social Dominance Orientation Scale (SDO). En: Robinson, J.; Shaver, P. \& Wrightsman, L. (Eds.) (1999). Measures of Political Attitudes. California: Academic Press.

Carrión, F. (2007). Percepción inseguridad ciudadana. Extraído el 28 de abril de 2008 de: http://www.flacso.org.ec/docs/ciudad_segura15.pdf

Cohen, J. (1988). Statistical Power Analysis for the Behavioral Sciences. Hillsdale, New Jersey: Lawrence Erlbaum Associates Publishers.

Cottam, M., Dietz-Uhler, B., Mastors, E. \& Preston, T. (2004). Introduction to Political Psychology. New Jersey: Lawrence Erlbaum Associates.

Crowson, H. M., Thoma, S. \& Hestevold, N. (2005). Is Political Conservatism Synonymous With Authoritarianism? The Journal of Social Psychology, 145, 5, 571-592.

Crowson, H. M. \& Debacker, T. (2006). The role of authoritarianism, perceived threat and need for closure or structure in predicting post - 9/11 attitudes and beliefs. The Journal of Social Psychology, 146, 733-750.

Cruz, J. M. (1999). Victimización por violencia urbana: niveles y factores asociados en ciudades de América Latina y España. Revista panamericana de salud pública, $5,4 / 5,259-267$.

Curbet, J. (2006). La globalización de la [in] seguridad. La Paz: Plural Editores.

Dammert, L. (2005). Violencia criminal y seguridad ciudadana en Chile. Santiago de Chile: Naciones Unidas.

Duckitt, J. (2001). A dual process cognitive-motivational theory of ideology and prejudice. En Zanna, M. (Ed.) (2001) Advances in experimental social psychology, 33. San Diego: Academic Press.

---------. (2006). Differential Effects of Right Wing Authoritarianism and Social Dominance Orientation on outgroup attitudes and their mediation by threat from and competitiveness to outgroups. Personality and Social Psychology Bulletin, $5,630-684$.

Duckitt, J. \& Sibley, C. (2007). Right Wing Authoritarianism, Social Dominance Orientation and the Dimensions of Generalized Prejudice. European Journal of Personality, 21, 113-130.

Revista Española de Investigación Criminológica

Artículo 4, Número 7 (2009) $\quad$ www.criminología.net

ISSN: $1696-9219$ 
Ferraro, K. (1995). Fear of Crime: Interpreting Victimization Risk. Nueva York: State University of New York

Grupo de Opinión Pública de la Universidad de Lima (GOP), (2007). I Encuesta Anual 2007 sobre la situación de la violencia en el Perú. Extraído el 20 de mayo de 2008 desde http://www.ulima.edu.pe/webulima.nsf/

Hammond, C. B. \& Calhoun, K. S. (2007). Labeling of Abuse experiences and rates of victimization. Psychology of Women Quarterly, 31, 4, 371-380.

Henry, P. J., Sidanius, J. Levin, S. \& Pratto, F. (2005). Social Dominance Orientation, Authoritarianism, and Support for Intergroup Violence Between the Middle East and America. Political Psychology, 26, 4, 569-583.

Instituto de Opinión Pública (IOP) de la Pontifica Universidad Católica del Perú (2008) El problema de la Inseguridad. Recuperado el 12 de febrero de 2009 desde http://www.pucp.edu.pe/iop/

James, B. (2006). The relationship between right-wing authoritarianism and attitudes toward violence: Further validation of the Attitudes toward Violence Scale. Social Behaviour and Personality, 34, 8, 923-926.

Jost, J., Glaser, J., Kruglanski, A. \& Sulloway, F. (2003). Political Conservatism as Motivated Social Cognition. Psychological Bulletin, 129, 3, 339-375.

Kerlinger, F. y Lee, H. (2002). Investigación del Comportamiento. México, D.F: McGraw-Hill.

Kohm, S. (2009). Spatial Dimensions of Fear in a High-Crime Community: Fear of Crime or Fear of Disorder? Revue canadienne de criminologie et de justice pénale, 51,1, 1-29.

Knight, K. (1999). Right Wing Authoritarianism Scale (RWA). En Robinson, J., Shaver, P. \& Wrightsman, L. (Eds.) (1999). Measures of Political Attitudes. California: Academic Press.

Leanne, S., Son Hing, D., Bobocel, R., Zanna, M. \& McBride, M. (2007). Authoritarian Dynamics and Unethical Decision Making: High Social Dominance Orientation Leaders and High Right-Wing Authoritarianism Followers. Journal of Personality and Social Psychology, 92, 67-81.

Mesch, G. (2000). Perceptions of Risk, Lifestyle activities, and Fear of Crime. Deviant Behavior, 2, 1, 47-63.

Moya, M. \& Morales-Marente, E. (2005). Reacciones psico-políticas ante los ataques terroristas del 11 de marzo de 2004. Revista de Psicología Social, 20, 331-350.

Muñoz, A. \& Instituto de Defensa Legal (IDEELE). (2008). Seguridad Ciudadana y presupuesto en el Perú. Extraído el 12 de febrero de 2009 desde http://www.seguridadidl.org.pe

Pratto, F., \& Shih, M. (2000). Social Dominance Orientation and Group Context in Implicit Group Prejudice. Psychological Science, 11, 6, 515-518.

Ramos, M. A. (2006). Masculinidades y violencia conyugal. Experiencias de vida de hombres de sectores populares de Lima y Cusco. Lima: Universidad Peruana Cayetano Heredia. (2008). La construcción de la sexualidad masculina. Violencia sexual. Clase magistral presentada en el Diploma de Psicología Forense y Penitenciaria, de la Facultad de Letras y Ciencias Humanas de la PUCP, Septiembre, Lima.

Sabucedo, J. M. (1996). Psicología Política. Madrid: Síntesis.

Sistema Nacional de Seguridad Ciudadana (SINASEC) \& APOYO Opinión y Mercado. (2005). Estudio de Victimización en las ciudades de Lima, Arequipa, Cusco,

Revista Española de Investigación Criminológica

Artículo 4, Número 7 (2009) $\quad$ www.criminología.net

ISSN: $1696-9219$ 
Huamanga, Iquitos y Trujillo. Extraído el 14 de febrero de 2009 desde http://www.seguridadidl.org.pe/

SYMANTEC (2004). The Internet Security Threat Report. Extraído el 27 de marzo de 2009 de www.symantec.com

Taylor, R. \& Hale, M. (1986). Testing alternative models of fear of crime. The Journal of Criminal Law \& Criminology, 77, 151-189.

Varat, J. (Comp.) (2007). Seguridad ciudadana en las Américas. Washington, D.C.: Woodrow Wilson International Center for Scholars.

Vozmediano, L., San Juan, C., \& Espinosa, A. (2006). Estimación del miedo al delito y escenarios de conducta: un estudio en la CAPV y Perú. En Corraliza, J. A., Berenguer, J. \& R. Martín (Eds.) (2006). Medio Ambiente, Bienestar Humano y Responsabilidad Ecológica. Madrid: Editorial Resma.

Vozmediano, L., San Juan, C. \& Vergara, A. (2008). Problemas de medición del miedo al delito. Algunas respuestas teóricas y técnicas. Revista Electrónica de Ciencia Penal y Criminología, 1-17.

Weinrath, M., Clarke, C. \& Forde, D. (2007). Trends in Fear of Crime in aWestern Canadian City: 1984, 1994, and 2004. Revue canadienne de criminologie et de justice pénale, $49,5,617-645$.

Yépez, E. (2004). Seguridad ciudadana: 14 lecciones fundamentales. Lima: Instituto de Defensa Legal (IDEELE).

Zaldívar, E. (2007). Conceptos de Victimología. Extraído el 12 de junio del 2008 desde http://www.cudi.edu.mx/salud2/proteccion_civil/victimologia.pdf 